If we invert the right half and place it in that position before the left eye in the stereoscope, it is seen double again; but in this case the sense of perspective is faulty. In some cases there is no difficulty in interpreting the view for it can be compared to the reflection of hills in the still waters of a lake. In other cases another interpretation is possible, as in the "six are seven cubes" illusion; we interpret the shadows of the various objects in a wholly different manner, and an absolutely new picture starts into view. Not all of the old view appears in the new. Which of the two views will predominate when the second half of the original picture is inverted and approximated to that in the stereoscope will depend on circumstances. If, as already stated, the inverted right half is placed in the stereoscope before the left eye and the inverted left half is brought into its proper position before the right eye, the view obtained is the water view. With the inverted left half before the left eye and the inverted right approximated thereto in the stereoscope the alternative view becomes prominent, depth and solidity being very pronounced. The parts of the original picture which are not essential to the new view apparently come forward, causing a relative movement of the new view backward. The parts which move forward may be bits from the fore or background; in some cases they appear like a painted screen between the observer and the distant view and in some, if not in all, cases can be blocked out without altering the new view.

According to this rendering of the facts, the foreground is not thrown into the distance and distant objects brought forward when the inverted and reversed halves of a stereoscopic picture are seen in a stereoscope; but a new view is seen in which the fusion sense has full play in the ordinary acceptation of the term.

As a consequence, the experiment does not support the contention that "Our fusion sense appears to be extremely elastic and readily capable of compromise or even suppression."

$$
\begin{gathered}
\text { Yours, etc., } \\
\text { T. Stewart Barrie, M.B., F.R.F.P.S.G. }
\end{gathered}
$$

13, Somerset Place,

GLASGOW, W.

20 February, 1919

\title{
RING INFILTRATION OF THE CORNEA IN PLAGUE
}

\section{To the Editor of The British Journal of Ophthalmology.}

SIR,-In the December, 1918, issue of the BRITISH Journal OF OPHTHALMOLOGY, page 612, Mr. D. V. Giri states that no case of acute general infection has been reported as a cause of ring 
infiltration of the cornea. In the metastatic cases recorded there has been a localized focus of sepsis in some other part of the body.

I believe that the condition is not rare in severe septicaemic plague. Recently I saw ring abscess well marked in both corneae of a female plague patient. When I first saw her she was lying semi-conscious on the fourteenth day of the disease. The corneal infiltration was then definite, but it was more so, and yellowish in tint, two days later. She died on the seventeenth day. There was no evidence of suppuration elsewhere; the only bubo was a moderately enlarged gland, the size of an almond, in the left groin.

In Bombay, years ago, I was struck with the very marked shrinkage of the flattened cornea, down to as little as $3 \mathrm{~mm}$., in atrophic eyeballs from plague endophthalmitis, seen at various intervals after recovery from the disease. Possibly, in these there may have been annular infiltration, which had resolved.

Yours, etc.,

H. HeRBERT, Lt.-Colonel, I.M.S.

\section{A NEW FORM OF BIFOCAL LENS}

To the Editor of The British Journal of Ophthalmology.

SIR,-Mr. Bardsley's ingenious scheme for solving the problem of the inclination of the lower segment of a bifocal combination is only of use for the limited case of those patients who are myopes and able to afford the very costly glass described by him. I wish to suggest that the problem may be attacked in another way. Percival, in his book, "The Prescribing of Spectacles," gives, on page 133, the necessary formulae for calculating the cylindrical effect of tilting a lens. A table might be drawn up from this showing the effect of tilting lenses of various strengths to an average reading inclination of $25^{\circ}$. All the surgeon would now require to do would be to refer to the table and prescribe a cylinder for the lower segment of the bifocal sufficient to neutralize the prismatic effect produced by the tilting. This method would be applicable to convex and concave lenses. I hope Mr. Bardsley, with the help of Dr. Fison, may consider the possibility of working out such a table. I must confess that I cannot trust my own mathematical talents to deal with the task.

I am, Sir, yours faithfully,

E. ERskine Henderson. 